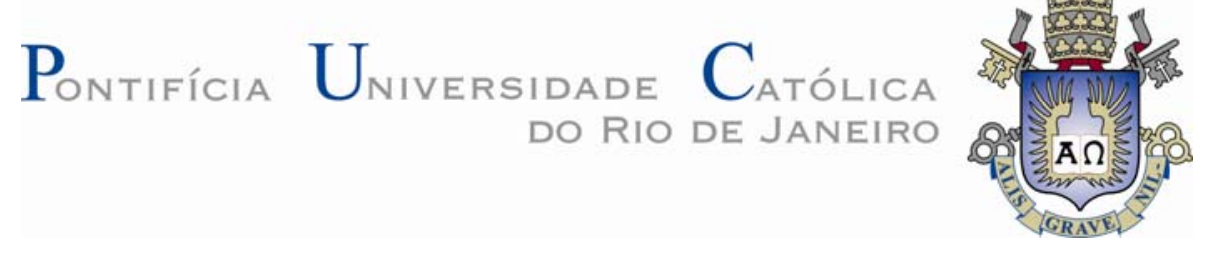

Luis Salomon Murillo Colquicocha

\title{
Avaliação de um Novo Método Para a Previsão da Pressão de Ruptura de Dutos que Contêm Colônias de Corrosão
}

Dissertação de Mestrado

Dissertação apresentada como requisito parcial para obtenção do título de Mestre pelo Programa de PósGraduação em Engenharia Mecânica da PUC-Rio.

Orientador: Jose Luiz França Freire 


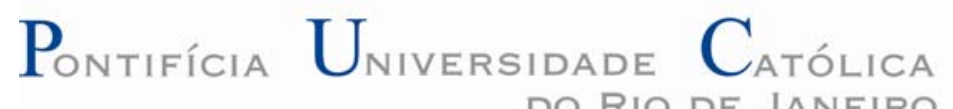

DO RIO DE JANEIRO

Luis Salomon Murillo Colquicocha

\section{Avaliação de um Novo Método Para a Previsão da Pressão de Ruptura de Dutos que Contêm Colônias de \\ Corrosão \\ Dissertação apresentada como requisito parcial para obtenção do título de Mestre pelo Programa de Pós- Graduação em Engenharia Mecânica da PUC-Rio. Aprovada pela Comissão Examinadora abaixo assinada.}

Prof. Jose Luiz França Freire

Orientador

Departamento de Engenharia Mecânica - PUC-Rio

Prof. Carlos Alberto de Almeida

Departamento de Engenharia Mecânica - PUC-Rio

Prof. Arthur Martins Barbosa Braga

Departamento de Engenharia Mecânica - PUC-Rio

Dr. Roberth Waldo Angulo Llerena

PUC-Rio

Prof. José Eugenio Leal Coordenador Setorial do Centro

Técnico Científico - PUC-Rio

Rio de Janeiro, 27 de abril de 2011 
Todos os direitos reservados. É proibida a reprodução total ou parcial do trabalho sem autorização da universidade, do autor e do orientador.

\section{Luis Salomon Murillo Colquicocha}

Graduou-se em Engenharia Mecânica na UNI (Universidad Nacional de Ingeniería), Lima-Peru, em 2005. Atua na área de Engenharia Mecânica, com ênfase em mecânica aplicada.

Ficha Catalográfica

Murillo Colquicocha, Luis Salomon

Avaliação de um Novo Método Para a Previsão da Pressão de Ruptura de Dutos que Contêm Colônias de Corrosão / Luis Salomon Murillo Colquicocha; orientador: Jose Luiz França Freire. - 2011.

149 f.: il.(color); $30 \mathrm{~cm}$

Dissertação (Mestrado) - Pontifícia Universidade Católica do Rio de Janeiro, Departamento de Engenharia Mecânica. Rio de Abril, 2011.

Inclui bibliografia.

1. Engenharia Mecânica - Teses. 2. Corrosão. 3. Colônias de defeitos. 4. Pressão de falha. 5. Elementos finitos. 6. Integridade estructural. I. Freire, Jose Luiz França. II. Pontifícia Universidade Católica do Rio de Janeiro. Departamento de Engenharia Mecânica. III. Título. 
Aos meus pais: Luis e Palmira e aos meus irmãos; responsáveis pela minha formação pessoal e profissional, e que me deixaram completamente à vontade para seguir os caminhos que considerei os melhores para min.

Sou eternamente grato. 


\section{Agradecimentos}

Ao meu orientador Prof. Jose Luiz França Freire, pelos ensinamentos, orientações, pela ajuda durante o tempo que estive no Brasil, pela paciência e por todas as formas de colaboração para a realização de minha dissertação de mestrado.

À PUC-Rio, pela oportunidade do curso de mestrado. Orgulho-me imensamente de ser parte dessa excelente instituição que contribui para o aperfeiçoamento intelectual, acadêmico e profissional de todos os seus alunos.

Ao Conselho Nacional de Desenvolvimento Científico e Tecnológico (CNPq) pelo apoio através do programa de financiamento de pesquisas científicas e tecnológicas nas diversas áreas do conhecimento.

A todos os professores pelos conhecimentos adquiridos, por suas aulas e pela forma que conduzem os alunos através do mestrado.

Ao suporte técnico ESSS pela ajuda na simulação numérica no ANSYS.

Ao meu amigo Americo Cunha pela ajuda e sugestões na revisão da dissertação.

Aos meus amigos e colegas, com os quais sempre compartilhei meus sucessos e fracassos, e que tornaram esta caminhada mais leve e tranquila, proporcionando inúmeros momentos de alegria e descontração.

À minha família que sempre esteve presente nos momentos de alegria e nas situações e decisões difíceis durante minha estadia no mestrado. 


\section{Resumo}

Luis Salomon Murillo Colquicocha. Avaliação de um Novo Método Para a Previsão da Pressão de Ruptura de Dutos que Contêm Colônias de Corrosão. Rio de Janeiro, 2011. 149p. Dissertação de Mestrado Departamento de Engenharia Mecânica, Pontifícia Universidade Católica do Rio de Janeiro.

Neste trabalho avalia-se uma metodología de cálculo para a previsão da pressão de falha de um duto que contem colônias de corrosão, chamada " Método das Linhas Ortogonais de Ruptura (MLOR)". As colônias de corrosão são representadas por defeitos retangulares, localizados na superfície externa do duto. O objetivo do MLOR é ser mais exato que os métodos semi-empíricos da ASME e DNV utilizados até o momento. O MLOR também tem como objetivo evitar resultados não conservativos que podem ser obtidos com o método Mixed Type of Interacting (MTI). O MLOR apresenta outra forma de avaliar a perda de espessura efetiva de um grupo de defeitos. Ele propõe que se tracem linhas representativas do caminho pelo qual a ruptura do duto tem maior possibilidade de ocorrer, usando linhas paralelas às direções longitudinal e circunferencial do duto. Estas linhas são chamadas de linhas ortogonais de ruptura (LOR). Também considera-se o emprego de um fator de resistência circunferencial f no cálculo de uma espessura efetiva para a colônia. Este fator multiplica somente os comprimentos das LOR que estiverem na direção circunferencial do duto. O material íntegro existente entre dois defeitos é considerado na resistência à ruptura por meio da seleção de um caminho crítico que une os defeitos retangulares e que usa as direções longitudinal e circunferencial. Para a avaliação do MLOR usou-se pressões de falha para tubos com colônias de defeitos determinados experimentalmente e já publicados, além de simulações numéricas feitas pelo método dos elementos finitos (MEF), utilizando-se para isto o software comercial ANSYS. Por fim, neste trabalho concluiu-se que o MLOR apresentou bons resultados (máximos erro médio igual a $-0.14 \%$ e desvio padrão do erro igual a $3.53 \%$ ) quando comparados com os resultados experimentais e numéricos, já publicados, de nove corpos de provas em tamanho real.

\section{Palavras-chave}

Corrosão; colônias de defeitos, pressão de falha; elementos finitos; integridade estrutural. 


\section{Abstract}

Luis Salomon Murillo Colquicocha. Evaluation of a New Method For Predicting Burst Pressure of Pipelines Containing Colonies of Corrosion. Rio de Janeiro, 2011. 149p. MSc. Dissertation - Departamento de Engenharia Mecânica, Pontifícia Universidade Católica do Rio de Janeiro.

This dissertation comprises the evaluation of a new calculation methodology developed to predict the burst pressure of pipeline segments containing corrosion colonies. The new methodology is called "Method of Orthogonal Lines Rupture (MLOR). The corrosion colonies are represented by rectangular defects, located on the outer surface of the pipeline segment. The objective of MLOR is to determine more accurate results than those calculated by the semi-empirical methods of ASME and DNV. The MLOR has also the objective of avoiding non-conservative results that can be obtained with the Mixed Type Interacting method (MTI). The MLOR presents a new way to assess the loss of effective thickness of a group of defects. It proposes the drawing of lines parallel to the longitudinal and circumferential directions that intend to represent the path by which the rupture of the pipeline is more likely to occur. These lines are called Orthogonal Lines of Rupture (LOR). It is also considered the employment of a strength circumferential factor $f$ in the calculation of the effective thickness of the colony. This factor multiplies only the lengths of the LOR which are associated with the circumferential direction of the pipeline. Using that, the intact material existing between two defects is considered in the calculation of the rupture resistance. The critical path connects the rectangular defects using lines parallel to the longitudinal and circumferential directions. The evaluation of the MLOR was accomplished by comparing its burst pressure calculations with already published results determined for nine full scale tests carried out with specimens that contained specially designed and constructed colonies of defects. The comparisons encompassed those experimentally published test results and also results determined by numerical simulations that employed the finite element method (ANSYS commercial software). Finally, the dissertation concludes that the MLOR showed good results (maximum average error equal to $-0.14 \%$ and standard deviation of the error equal to 3.53\%) compared with experimental and numerical results.

\section{Keywords}

corrosion; groups of defects; burst pressure; finite elements; structural integrity. 


\section{Sumário}

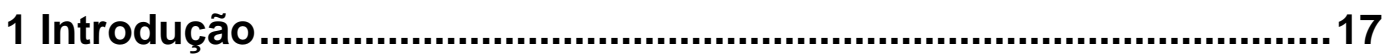

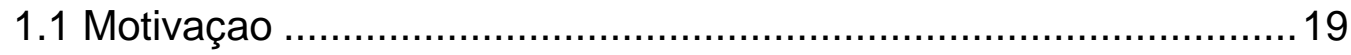

1.2 Objetivos do Trabalho .......................................................... 19

1.2.1 Objetivo Geral ....................................................... 19

1.2.2 Objetivos Específicos .................................................. 19

1.3 Revisão Bibliográfica .................................................... 20

1.4 Organização do Trabalho ...............................................23

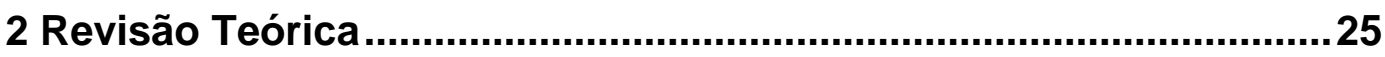

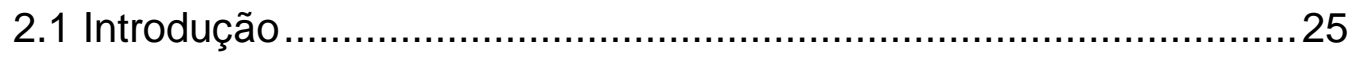

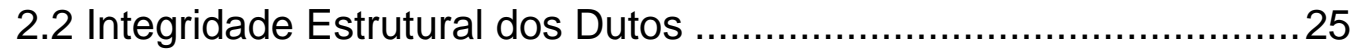

2.3 Definição e Tipos de Defeitos de Corrosão ..................................26

2.3.1 Interação de Defeitos e seus Tipos .....................................27

2.4 Avaliação de Defeitos por Níveis de Complexidade .......................30

2.5 Métodos Analíticos Semi-Empíricos ....................................... 32

2.5 .1 Introdução ............................................................ 32

2.5.2 Tensões Atuantes Numa Casca Fina Submetida à Pressão Interna ................................................................................... 34

2.5.2.1 Tensões Atuantes em Dutos sem Defeitos de Corrosão ...34

2.5.2.2 Tensões Atuantes em Dutos com um Defeito de Corrosão 35

2.5.3 Métodos Tradicionais de Cálculo de Pressão de Falha ...........37

2.5.3.1 Método ASME B31G ............................................ 38

2.5.3.2 Método RSTRENG 085dL ou ASME B31G Modificado.....39

2.5.3.3 Método DNV RP-F101 para Defeitos Únicos ....................40 40

2.5.3.4 Análise Algébrica da Equação da DNV RP-F101 ............40 40

2.6 Método Mixed Type of Interacting (MTI) ................................44

2.6.1 Procedimento de Cálculo do Método MTI ..........................45

2.6.2 Limitações do Método $\mathrm{MTI}$............................................47

2.7 Método das Linhas Ortogonais de Ruptura (MLOR).................... 48 
2.7.1 Procedimento de Cálculo do Novo Método MLOR …................ 48

2.7.2 Análise do Método das Linhas Ortogonais de Ruptura .............53

2.7.3 Fator $\mathrm{f}$ Utilizado no Cálculo da Perda de Espessura Efetiva ....57

2.7.4 Análise das Tensões para Dois Defeitos.................................60

3 Modelagem Numérica de Defeitos de Corrosão em Dutos...............64

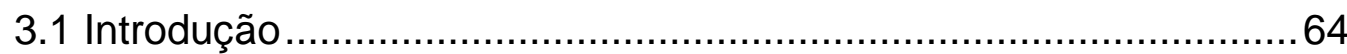

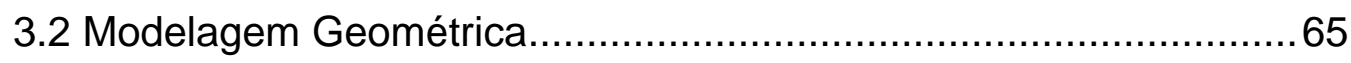

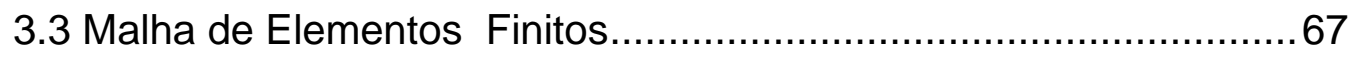

3.4 Condições de Contorno e Carregamentos.......................................69

3.5 Material e suas Propriedades ..................................................... 72

3.6 Considerações Sobre os Incrementos de Carga Utilizados .............76

3.7 Considerações Sobre o Critério de Ruptura e sua Relação com a

Falha Numérica ............................................................................ 77

4 Resultados: Análise Não-Linear de Dutos com Defeitos de

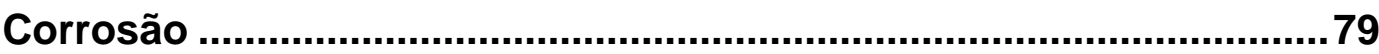

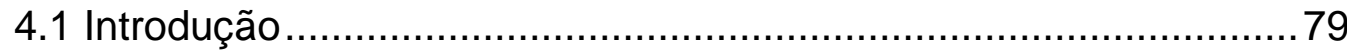

4.2 Resultados e Comparação entre os Métodos .................................. 79

4.3 Resultados das Simulações Numéricas............................................ 80

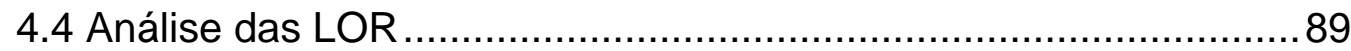

4.5 Análise Geral quanto aos Métodos de Cálculo ...............................94

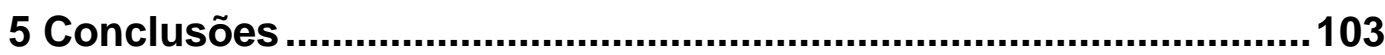

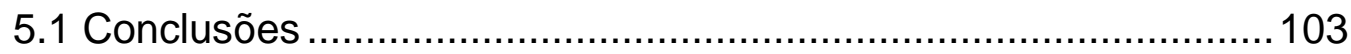

5.2 Recomendações para Trabalhos Futuros.................................... 105

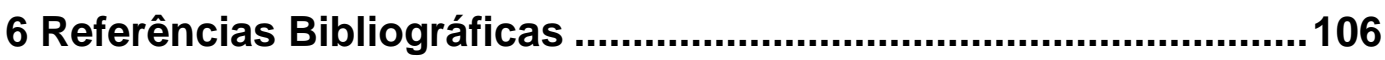

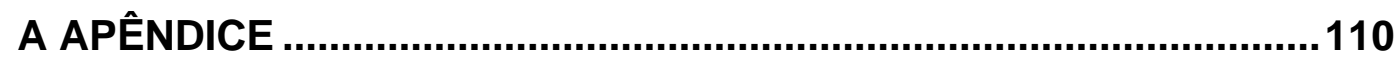

A.1 LOR para o Espécime IDTS12 ……....................................... 110

A.2 Previsão das Pressões Segundo o Método MLOR ……………... 112

A.3 Fator de Resistência Remanescente C ..................................... 114

A.4 Tensões de von Mises para a Primeira e Última Camada.............116 


\section{Lista de figuras}

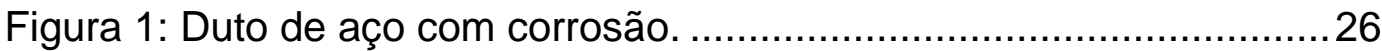

Figura 2: Exemplos de tipos de defeitos de corrosão (fonte: http://www.portaldagalvanizacao.com.br/corrosao.asp - novembro/2010)27

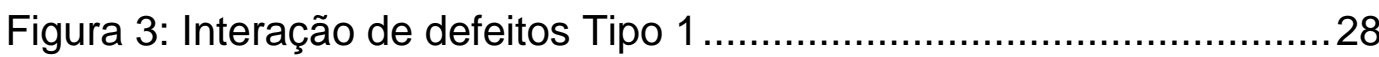

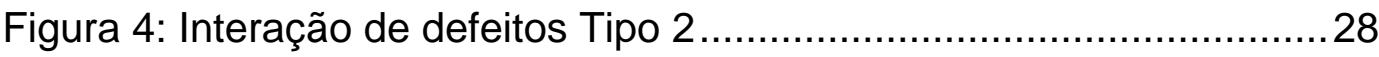

Figura 5: Interação de defeitos Tipo 1, 2 e 3 ........................................... 29

Figura 6: Exemplo da interação Mista ................................................... 29

Figura 7: Vaso de pressão submetido à pressão interna (Fonte: Freire

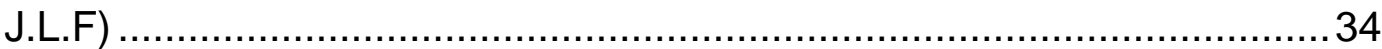

Figura 8: Vaso de pressão com um defeito base .....................................37

Figura 9: Fator de resistência remanescente (C) vs fator de folias (M) ....42

Figura 10: Fator de resistência remanescente (C) vs (d/t) ......................43

Figura 11: Vaso de pressão com dois defeitos interagindo ....................... 45

Figura 12: Condições de traçado da LOR ..............................................49

Figura 13: Exemplo teórico do MLOR com dois defeitos .........................50

Figura 14: Exemplo teórico do MLOR com três defeitos ...........................51

Figura 15: Análise da influência do comprimento longitudinal (s).............53

Figura 16: Análise da influência do comprimento circunferencial (c)........54

Figura 17: Análise da influência do comprimento circunferencial (c)........55

Figura 18: Comportamento do fator de resistência remanescente ............56

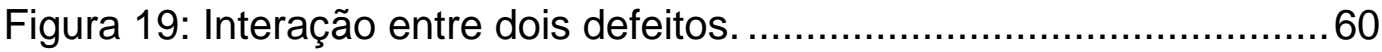

Figura 20: Variação fator de resistência remanescente (C) ......................62

Figura 21: Variação fator de resistência remanescente com o fator f. .....63

Figura 22: Modelagem geométrica de apenas 1/2 do vaso de pressão

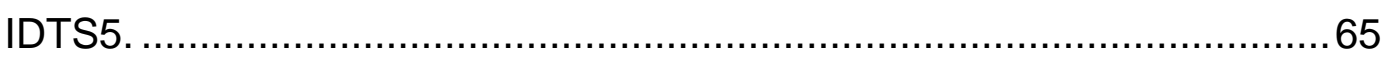

Figura 23: Parâmetros necessários para modelagem do defeito .............66

Figura 24: Tipo de elemento finito hexaédrico SOLID45 e SOLID95 [27] 67 Figura 25: Transição com a malha não estruturada na região próxima do 
defeito.

Figura 26: Transição com a malha estruturada na região próxima do defeito.

Figura 27: Condições de contorno e carregamento impostos no duto. ....71

Figura 28: Condição de simetria para os espécimes IDTS. 71

Figura 29: Tensão verdadeira versus deformação verdadeira (API 5L $\mathrm{X} 80)$ 73

Figura 30: Distribuição das tensões de VM do espécime IDTS5...............81

Figura 31: Faixa das tensões de VM do espécime IDTS5 …...................82

Figura 32: Tensões de VM da superfície exterior ou primeira camada ....83

Figura 33: Tensões de VM da superfície interior ou ultima camada..........84 84

Figura 34: Tensões de von Mises na camada interna ..............................84

Figura 35: Superfície das tensões de von Mises...................................... 85

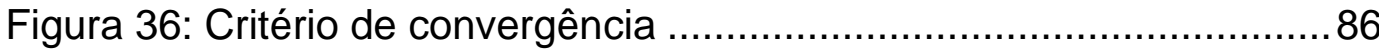

Figura 37: Critério de convergência última camada de elementos 86

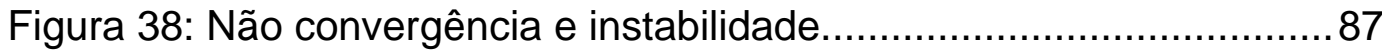

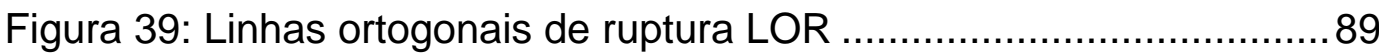

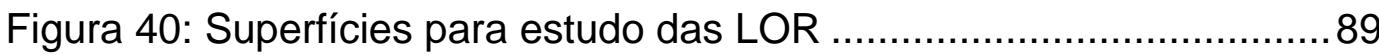

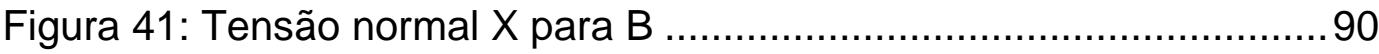

Figura 42: Tensão normal Y para B ....................................................90

Figura 43: Tensão normal Z para B.................................................. 91

Figura 44: Tensão cisalhante XZ para B ………................................91

Figura 45: Tensão cisalhante XY para B ............................................ 91

Figura 46: Tensão cisalhante YZ para B .............................................. 92

Figura 47: Tensão principal (1) para B ………….............................92

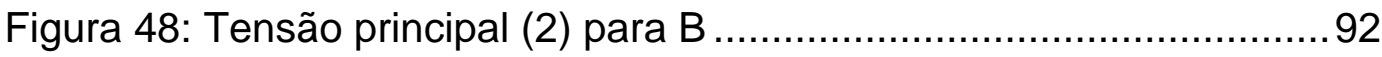

Figura 49: Tensão principal (3) para B …….......................................93

Figura 50: Tensão de von Mises para B..............................................

Figura 51: Razões entre as pressões previstas e a pressão de teste ......94

Figura 52: Razões entre as pressões para o MTI, MLOR e MEF.............95 


\section{Lista de tabelas}

Tabela 1: Dimensões reais dos espécimes tubulares e dos defeitos

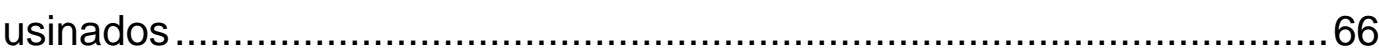

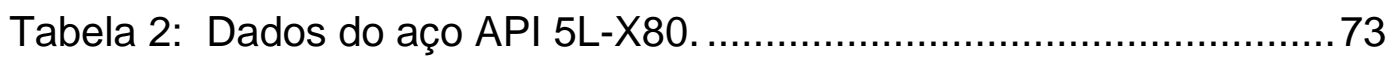

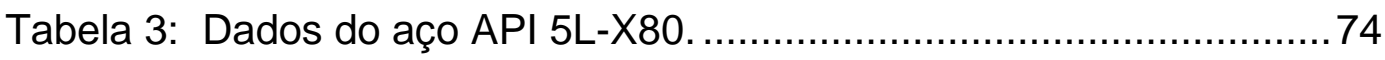

Tabela 4: Previsão da pressões de falha dos casos estudados................80

Tabela 5: Erros das pressões de falha ................................................ 80

Tabela 6: Resultados da simulação numérica......................................... 88

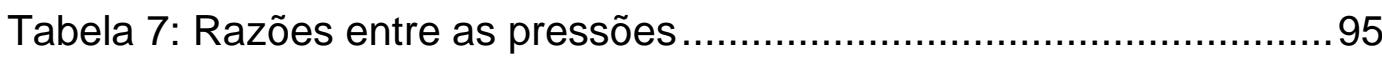

Tabela 8: Resultados segundo o método MLOR................................. 113 


\section{Lista de quadros}

Quadro 1: Processo geral da análise pelo MEF 64

Quadro 2: Comparação entre resultados experimentais e numéricos

(MEF) para os espécimes IDTS 2 a 7

Quadro 3: Comparação entre resultados experimentais e numéricos

(MEF) para os espécimes IDTS 8 a 12

Quadro 4: Comparação entre resultados experimentais e analíticos (MLOR)

Quadro 5: Comparação entre resultados experimentais e analíticos (MLOR)

Quadro 6: Tensões de von Mises para os espécimes IDTS 2 a $7 \quad 101$

Quadro 7: Tensões de von Mises para os espécimes IDTS 8 a 12102

Quadro 8: Grupos de defeitos para o espécime IDTS-12 


\section{Lista de símbolos}
$A_{o}$
Área longitudinal do duto sem corrosão
$\varepsilon_{C}$
Deformação circunferencial
$\varepsilon_{L}$
Deformação longitudinal ou axial
$\varepsilon_{R}$
Deformação radial
A
Parâmetro representativo da área longitudinal com corrosão
$p_{0} \quad$ Pressão de falha para o duto de parede fina sem corrosão.
$\sigma_{C} \quad$ Tensão circunferencial
$\sigma_{V M} \quad$ Tensão de von Mises
$\sigma_{L} \quad$ Tensão longitudinal ou axial
$\sigma_{R} \quad$ Tensão radial
$\sigma_{\text {Tresca }} \quad$ Tensão de Tresca
C Fator de resistência remanescente
c Distância circunferencial entre os defeitos
D Diâmetro externo do duto.
d Profundidade de perda de espessura do defeito.
$d_{g} \quad$ Perda de espessura efetiva do grupo de defeitos 
$d_{g} \quad$ Profundidade de perda de espessura do grupo de defeitos.

$D_{i} \quad$ Diâmetro interno do duto.

$d_{i} \quad$ Profundidade de perda de espessura do defeito $(i=1,2 \ldots)$.

f $\quad$ Fator de resistência circunferencial.

L $\quad$ Comprimento longitudinal do defeito.

Lg Comprimento do grupo de defeitos

M Fator adimensional de forma de Folias

$p \quad$ Pressão interna do duto.

$p_{f} \quad$ Pressão de falha para o duto com defeito de corrosão.

Distância longitudinal entre os defeitos

S Resistência a colapso plástico.

$S_{u} \quad$ Limite de resistência à tração do material.

S Limite de resistência à tração verdadeiro do material.

Sy Limite de escoamento do material.

S Limite de escoamento verdadeiro do material.

$t \quad$ Espessura do duto.

Vg Volume de perda de metal causado pela corrosão

W Comprimento circunferencial ou largura do defeito.

Wg Largura do grupo de defeitos 


\section{Abreviatura}

APDL ANSYS Parametric Design Language

API The American Petroleum Institute

ASME American Society of Mechanical Engineers

CAD Computer Aided Design

CAE $\quad$ Computer Aided Engineering

CNPq Conselho Nacional de Desenvolvimento Científico e Tecnológico

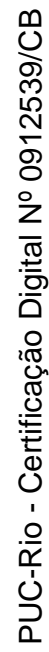

DNV Det Norske Veritas

DNV Det Norske Veritas

EUA Estados Unidos da América

FEM Finite Element Method

IDTS Interactive Defect Tubular Specimen

LOR Linhas Ortogonais de Ruptura

MEF $\quad$ Método dos Elementos Finitos

MFL Magnetic Flux Leakage

MLOR Método das Linhas Ortogonais de Ruptura

MTI Mixed Type of Interaction Method

PIG Pipeline Inspection Gadget

SMTS Specified Minimum Tensile Strength

SMYS Specified Minimum Yield Strength 